\title{
APUNTES PARA LA MEJORA DE LOS CONTENIDOS CONCEPTUALES DEL CURSO INSTITUCIONES DE DERECHO PRIVADO (PUCP)
}

\section{Notes for the improvement of the conceptual contents of the Instituciones de Derecho Privado course (PUCP)}

Héctor Campos García*

Pontificia Universidad Católica del Perú

Profesor de Derecho Privado de la Pontificia Universidad Católica del Perú y en la Universidad de Lima. Magíster en Derecho con mención en Derecho Civil y Comercial por la Universidad Nacional Mayor de San Marcos. Integrante de la Delegación Peruana en el Grupo para la Armonización del Derecho en América Latina (GADAL). Asociado Senior en Linares Abogados. ORCID iD: 0000-0002-4032-4419. Contacto: h.campos@pucp.edu.pe 


\section{Resumen:}

El presente documento analiza los contenidos conceptuales del curso Instituciones de Derecho Privado en el Nuevo Plan de Estudios de la Facultad de Derecho de la Pontificia Universidad Católica del Perú. Se abordan dos problemas. En primer lugar, las posibles superposiciones con los contenidos conceptuales de otros cursos del Nuevo Plan, tales como Introducción a las Ciencias Jurídicas o Derechos Fundamentales e Interpretación Constitucional. En segundo lugar, el conjunto de temas que no se desarrollan a pesar de formar parte de las unidades temáticas del curso. Finalmente, se formulan recomendaciones para afrontar los problemas mencionados.

\section{Abstract:}

This document analyzes the conceptual contents of the Private Law Institutions course in the New Study Plan of the Faculty of Law of the Pontificia Universidad Católica del Perú. Two problems are addressed. Firstly, the possible overlaps with the conceptual contents of other courses of the New Plan such as Legal Science Introduction or Fundamental Rights and Constitutional Interpretation. Secondly, the set of topics that are not developed despite being part of the thematic units of the course. Finally, recommendations are made to deal with the aforementioned problems.

\section{Palabras clave:}

Formación por competencias - Contenidos conceptuales - Instituciones del Derecho Privado - Introducción a las ciencias jurídicas - Facultad de Derecho PUCP

\section{Keywords:}

Formation by competences - Conceptual content - Institutions of private law - Legal science introduction - PUCP Law School

\section{Sumario:}

1. A modo de introducción: objetivo y límites de la presente reflexión - 2. Diagnóstico preliminar de los contenidos conceptuales en el curso de IDP y de la forma como son abordados - 3. Observaciones a los contenidos conceptuales del curso IDP y su implementación en el Nuevo Plan - 4. Sugerencias para un rediseño de los contenidos conceptuales del curso IDP - 5. Reflexión final: el carácter propedéutico de las IDP - 6 . Recomendaciones: propuesta de contenidos conceptuales para el curso IDP - 7. Lista de referencias 


\section{A MODO DE INTRODUCCIÓN: OBJETIVO Y LÍMITES DE LA PRESENTE REFLEXIÓN}

El autor de esta reflexión inició sus estudios en la Facultad de Derecho de la Pontificia Universidad Católica del Perú (en adelante, PUCP) en el año 2005. En dicho momento se encontraba vigente el Plan de Estudios de 2001, el cual ordenaba los cursos del Área de Derecho Civil (obligatorios) de la siguiente forma:

\begin{tabular}{|l|l|}
\hline \multicolumn{1}{|c|}{ Ciclo } & \multicolumn{1}{c|}{ Cursos } \\
\hline Primero & Derecho Civil 1: PP.GG y PP.NN. \\
\hline Segundo & $\begin{array}{l}\text { Derecho Civil 3: Reales } \\
\text { Personas Jurídicas } \\
\text { Derecho Civil 4: Acto Jurídico }\end{array}$ \\
\hline Tercero & $\begin{array}{l}\text { Derecho Civil 5: Obligaciones } \\
\text { Derecho Civil 6: Familia }\end{array}$ \\
\hline Cuarto & Derecho Civil 8: Contratos \\
\hline Quinto & $\begin{array}{l}\text { Contratos Especiales 1 } \\
\text { Derecho Civil 11: Sucesiones }\end{array}$ \\
\hline Sexto & Derecho Internacional Privado \\
\hline
\end{tabular}

Tabla 1. Fuente: Elaboración propia.

Claramente, la opción que se había tomado en el momento de articular la malla de los cursos universitarios en el Plan de Estudios de 2001 coincide con los Libros que conforman el Código Civil peruano ${ }^{1}$ (en adelante, CC), tal como sucedía con los Planes de Estudios previos², lo cual pone de manifiesto

1 El Código Civil peruano vigente (1984) consta de un Título Preliminar, diez Libros y un Título Final. Los Libros son los siguientes: Derecho de las Personas, Acto Jurídico, Derecho de Familia, Derecho de Sucesiones, Derechos Reales, Las Obligaciones, Fuente de las Obligaciones, Prescripción y Caducidad, Registros Públicos, Derecho Internacional Privado.

2 En el Plan de Estudios de 1993 la situación era equivalente, salvo por el hecho que el curso Contratos Especiales era denominado como: Derecho Civil 9: Contratos típicos 1. En el Plan de Estudios de 1981, bajo la vigencia del Código Civil peruano de 1936 (compuesto por un Título Preliminar, Libro Primero: Del derecho de las persona, Libro Segundo: Del derecho de familia, 
una tendencia arraigada en la formación sobre Derecho Civil en la Facultad de Derecho de la PUCP, cuyos efectos en la segmentación del conocimiento han sido advertidos (León, 2012, p. 8).

En el año 2015, la Facultad de Derecho de la PUCP implementó un nuevo Plan de Estudios (en adelante, el Nuevo Plan) lo cual significó asumir un enfoque curricular orientado a la construcción de competencias (Sanromán y Morales, 2016, pp. 180-201), en el espíritu del Modelo Educativo PUCP³, según el cual "se propone servir al país y al mundo mediante la formación integral y humanista de los estudiantes, el desarrollo del conocimiento y nuestra proyección hacia la sociedad" (PUCP, 2016, p. 7).

Como consecuencia de lo anterior, se produjo la reestructuración de los sílabos ${ }^{4}$ de los cursos de la malla curricular, de forma que se identificaron las competencias ${ }^{5}$ a las que cada curso aportaba; así como los resultados de apren-

Libro Tercero: Del derecho de sucesión, Libro Cuarto: De los derechos reales, Libro Quinto: Del derecho de obligaciones y Disposiciones Finales), la situación no era diferente: Primer semestre (Derecho Civil I: Principios Generales y Personas Naturales), Segundo semestre (Derecho Civil II: Responsabilidad Civil, Derecho Civil III: Derechos Reales, Derecho Civil IV: Acto Jurídico), Tercer semestre (Derecho Civil V: Obligaciones), Cuarto semestre (Derecho Civil VI: Contratos, Derecho de Familia), Quinto semestre (Garantías, Contratos II: Contratos especiales) y Sexto semestre (Derecho Civil VII: Contratos especiales, Derecho Civil VIII: Sucesiones, Derecho Internacional Privado). Un recuento de los Planes de Estudio de la Facultad de Derecho de la PUCP se puede encontrar en Ramos y Cuadros (2019, pp. 318-332).

3 El Modelo Educativo PUCP (PUCP, 2016) se puede consultar en el siguiente enlace: https://www. pucp.edu.pe/documento/modelo-educativo-pucp/

$4 \quad$ Según la Guía para la elaboración del Plan de Estudios de Pregrado, el sílabo es el documento oficial que señala el aporte del curso al logro de una o más competencias del perfil del egresado, en el cual se consignan los resultados de aprendizaje por lograr, los contenidos, la metodología que se implementará para alcanzar tales logros y la forma en que se evaluará (PUCP, 2014, p. 31).

5 La competencia es entendida como "la capacidad de desempeñarse en contexto haciendo uso de saberes de distinta naturaleza-conceptuales, procedimentales y actitudinales-" (Car rillo y Gálvez, 2017, p. 9). Las competencias genéricas del alumno que concluye sus estudios de pregrado en la PUCP son las siguientes: (i) Aprendizaje autónomo: gestiona su proceso de aprendizaje de manera autónoma y permanente; (ii) Ética y ciudadanía: actúa con responsabilidad ética y ciudadana, reconociendo y respetando la diversidad, la autonomía y la dignidad de los demás; (iii) Comunicación eficaz: comunica eficazmente ideas con claridad, coherencia y consistencia, usando un lenguaje formal, oral o escrito; (iv) Razonamiento lógico-matemático: utiliza el razonamiento lógico-matemático para interpretar información o solucionar problemas académicos y de la vida cotidiana; (v) Investigación: investiga de manera crítica, reflexiva y creativa y presenta formalmente sus resultados; (vi) Trabajo en equipo: trabaja colaborativamente en equipos disciplinarios y pluridisciplinarios; y, (vii) Participación en proyectos: contribuye en el diseño e implementación de proyectos que aporten responsablemente al desarrollo social, 
dizaje ${ }^{6}$ de cada uno de ellos, los cuales se obtienen a través del desarrollo de contenidos conceptuales, procedimentales y actitudinales. Necesariamente, esta perspectiva supone un abandono de la práctica, antes mencionada y propiciada por el antiguo plan de estudios, de diseñar los cursos de Derecho Civil a través de los Libros del Código Civil, por ejemplo.

Dado dicho contexto, el objetivo de la presente reflexión es realizar algunos aportes puntuales que pueden contribuir a la revisión de los contenidos conceptuales del curso de Instituciones del Derecho Privado (en adelante, IDP), el cual se constituye en el primer curso obligatorio de los que componen la saga del Derecho Civil, en atención a su importancia dentro de la formación de las y los estudiantes.

La revisión de los contenidos conceptuales de IDP se realiza con ocasión a que el autor asume, en el ciclo 2020-1, la impartición del referido curso en la Facultad de Derecho de la PUCP7 . En ese sentido, haber estado a cargo de los cursos de Instituciones del Derecho Privado 2, Obligaciones y Responsabilidad Civil en la misma casa de estudios, permite realizar una evaluación de

ambiental, cultural o científico (PUCP, 2016, p. 42). Las competencias específicas de Derecho son las siguientes: (i) Marco ético de la profesión: (el egresado) conoce, se identifica con el marco ético profesional, reconoce sus implicancias prácticas y lo aplica en su ejercicio profesional, (ii) Excelencia académica: (el egresado) domina el sistema jurídico peruano (orígenes y fuentes; doctrina; instituciones y jurisprudencia) y aplica sus postulados en el ejercicio de su profesión, (iii) Relación con el entorno profesional: (el egresado) conoce la estructura y el funcionamiento de las organizaciones nacionales e internacionales y se desenvuelve eficazmente en ellas y con respecto a ellas, (iv) Lógica jurídica: (el egresado) domina y aplica los principios de la lógica y la argumentación jurídica para analizar y resolver un caso, (v) Paradigma del Estado Constitucional de Derecho: (el egresado) conoce y aplica los principios del Estado Constitucional de Derecho y los Derechos Fundamentales; y, (vi) Cumplimiento de tareas: (el egresado) desarrolla sus tareas de modo eficiente, con iniciativa, creatividad y organización. Estas competencias específicas se pueden consultar en el siguiente enlace: https://facultad.pucp.edu.pe/derecho/wp-content/ uploads/2014/10/Competencias-criterios-y-resultados-de-aprendizaje.pdf

6 Según indican Carrillo y Gálvez, el resultado de aprendizaje es un "logro específico, que se caracteriza por ser observable o verificable, al que deben arribar los estudiantes al finalizar un curso de su plan de estudios" (2017, p. 34). La relevancia actual de los resultados de aprendizaje (learning outcomes) en el proceso de aprendizaje, si bien tiene sus antecedentes en los trabajos de Ivan Pavlov (1849-1936) y los desarrollos John B. Watson (1878-1958) y Burrhus Frederic Skinner (1904-1990), se vincula con los Acuerdos de Bolonia (2003) (Adam, 2006, pp. 2-4). Los desafíos en cuanto al desarrollo e implementación de los resultados de aprendizaje han sido reseñados por Kennedy, Hyland y Ryan, 2007.

7 Conforman el equipo del curso de IDP en el ciclo 2020-1: Sebastián Paz, Sergio Gonzalez, Esther Zagaceta y Christian Toribio. En el ciclo 2021-1 se sumó al equipo: Darleen Vicente. 
los contenidos conceptuales de IDP a la luz de su funcionalidad a lo largo de los cursos posteriores de Derecho Civil Patrimonial.

Una advertencia debe ser realizada. Dada la estructura del Nuevo Plan, el diagnóstico, observaciones y recomendaciones que se formulen a continuación se tienen que entender exclusivamente aplicables a la Facultad de Derecho de la PUCP. Si bien los contenidos o saberes conceptuales de IDP pueden coincidir con otros cursos en otras Facultades de Derecho del Perú, no es posible sostener que aquello que se señale a continuación puede ser generalizado a otras Facultades de Derecho que tienen sus propias particularidades y características.

\section{DIAGNÓSTICO PRELIMINAR DE LOS CONTENIDOS CONCEPTUALES EN EL CURSO DE IDP Y DE LA FORMA COMO SON ABORDADOS}

Antes de indicar las observaciones respecto a los contenidos conceptuales del curso IDP, es indispensable realizar un breve diagnóstico respecto de la forma en la que se abordan dichos contenidos ${ }^{8}$ del referido curso.

Para tal fin, se ha tomado el sílabo del curso IDP, el cual es de acceso libre; así como los apuntes de alumnos que han cursado IDP entre los ciclos 2016-1 y 2020-2 a los cuales se ha podido tener acceso. En relación con estos últimos documentos, no se ha considerado el primer año de implementación del Nuevo Plan, para así centrar el análisis en un período en el que el curso, con su nueva estructura, pueda considerarse como maduro.

Tres aspectos ameritan ser destacados en el citado diagnóstico. En primer lugar, la Facultad de Derecho ha apostado por iniciar la formación en Derecho Privado de sus estudiantes con el desarrollo y construcción de una Parte General (Morales, 2010; Mendoza, 2018), lo cual es coherente con una articulación, como se ha indicado (Mengoni, 2011a, p. 309), de tipo empírico-descriptiva, lógico-analítica y lógica-sistemática, propia de una Facultad de Derecho. Ello se pone en relieve desde el momento en que se dejó de lado la secuencia Derecho Civil 1: Principios generales y persona naturales y Derecho Civil 4: Acto Jurídico, que respondía a una enseñanza del Derecho Civil a partir del CC; para dar paso al binomio IDP e Instituciones del Derecho Privado 2 (en adelante, IDP2) para

$8 \quad$ La presente reflexión se circunscribe a los contenidos conceptuales. No se hace alusión alguna a los contenidos procedimentales y actitudinales. Sobre el particular, para percibir la diferencia entre los tipos de contenidos antes mencionados se puede revisar: Carrillo y Gálvez (2017, pp. 36-43).

9 Puede accederse al sílabo en el siguiente enlace: https://internacionalizacion.pucp.edu.pe/ wp-content/uploads/2016/10/1-DEC280-Instituciones-del-Derecho-Privado.pdf 
un tratamiento institucional de las bases del Derecho Privado que es uno de los retos de su enseñanza (León, 2012, p. 8).

En segundo lugar, el curso de IDP si bien es considerado como equivalente curricular ${ }^{10}$ al curso Derecho Civil 1 (perteneciente al Plan de Estudios del 2001$1)$, no lo es a nivel de la determinación de los contenidos conceptuales ${ }^{11}$. De este modo, según la sumilla de IDP ${ }^{12}$, los aspectos conceptuales a desarrollar son los siguientes: (i) principios y fuentes del derecho civil (sic), (ii) personas naturales, (iii) personas jurídicas, (iv) situaciones jurídicas. De hecho, en el sílabo vigente del curso de identifican cuatro unidades temáticas que son las siguientes: (i) introducción a las Instituciones del Derecho Privado, (ii) el concebido y la persona natural, (iii) la persona jurídica como sujeto de derecho y, (iv) situaciones y relaciones jurídicas.

En tercer lugar, la forma de abordar los contenidos conceptuales de IDP se puede agrupar en dos grandes tendencias: (i) aquella que continúa desarroIlando, sustancialmente, los mismos contenidos conceptuales que en Derecho Civil 1; y, (ii) aquella que desarrolla los contenidos conceptuales de IDP según el Nuevo Plan. Dentro de esta última tendencia es posible identificar dos ulteriores aproximaciones. Una que es proclive al análisis, en ese orden, del Título Preliminar y del Libro Primero (Personas) del CC, y otra que aborda los temas, pero desde una perspectiva institucional sin limitarse a la estructura del CC.

Un primer balance del diagnóstico, apenas efectuado, permite sostener que, si bien la Dirección de Estudios ha promovido el enfoque programa a través de la elaboración de sílabos comunes, lo cual ha permitido avances

10 La equivalencia curricular de IDP y de Derecho Civil 1, así como la de todos los cursos del Nuevo Plan se encuentran en el siguiente link: http://facultad.pucp.edu.pe/derecho/wp-content/ uploads/2014/10/2019-01-21.Equivalencias-actualizadas.pdf

11 El curso de Derecho Civil 1 se limitaba al estudio de dos grandes unidades temáticas: (i) las instituciones presentes en el Título Preliminar del Código Civil y (ii) el derecho del concebido y las personas naturales.

12 La sumilla del curso IDP dispone lo siguiente: "Instituciones de Derecho Privado introduce al alumno en las instituciones y conceptos básicos del derecho civil y se imparte en el primer ciclo. Se desarrolla en sesiones principalmente teóricas que comprende el análisis de doctrina, legislación y jurisprudencia nacional y extranjera. Busca que el estudiante conozca las principales instituciones jurídicas que regulan las relaciones entre privados, por lo que contribuye al desarrollo de la competencia excelencia académica. El curso aborda los conceptos fundamentales del derecho privado relativos a las personas naturales, personas jurídicas y situaciones jurídicas. Esto también implica el estudio de los principios y las fuentes del derecho civil". Esta sumilla, así como la de todos los cursos obligatorios, se encuentra disponible en el siguiente link: http:// facultad.pucp.edu.pe/derecho/wp-content/uploads/2019/03/Sumillas-de-los-cursos-obligatorios.WEB_.pdf 
en la ejecución del Nuevo Plan, aún no existe uniformidad en los contenidos conceptuales de IDP, lo cual tiene una incidencia directa con la articulación horizontal de los siguientes cursos vinculados al Derecho Civil y, en general, a los demás cursos del Nuevo Plan ${ }^{13}$.

No está demás precisar que la divergencia indicada no tiene ninguna relación con la artificial diferencia metodológica entre una enseñanza teórica y una enseñanza casuística (Lipari, 2004, p. 1391), y que mucho menos tiene que ver con la contraposición entre la enseñanza legal del civil law y common law (Merryman, 1975, pp. 168-194; Genty, 2018, p. 1), sino que es un aspecto que para ser superado requiere de la necesaria cooperación que debe darse entre docentes, entre docentes y estudiantes; y entre los propios estudiantes en todo proceso de aprendizaje, en pro de lograr conseguir el perfil de egreso en la formación jurídica que brinda la PUCP.

A continuación, se formulará las principales observaciones respecto de los contenidos conceptuales del curso de IDP, las cuales han sido el resultado de confrontar los documentos a los que se ha hecho alusión al inicio de la presente sección.

\section{OBSERVACIONES A LOS CONTENIDOS CONCEPTUALES DEL CURSO IDP Y SU IMPLEMENTACIÓN EN EL NUEVO PLAN}

Las observaciones a los contenidos conceptuales de IDP son de dos órdenes. En primer lugar, en la posible superposición de contenidos con otros cursos de la malla curricular del Nuevo Plan. En segundo lugar, aquellos posibles contenidos conceptuales que no reciben tratamiento en IDP, aunque a juicio del autor, podrían recibirlo.

En ambos extremos se alude únicamente a una posibilidad, ya que se parte del hecho que la materia prima en la que se ha basado el presente análisis (sílabo y apuntes de alumnos) no reflejan la realización del nivel micro curricular del programa de la formación del abogado PUCP, pues también debe evaluarse el

13 El programa de formación del abogado PUCP está compuesto por tres niveles; a saber: (i) nivel macrocurricular (se refiere a los lineamientos educativos principales y las metas formativas; se expresa en el perfil del egresado de la facultad, el cual se enmarca en el modelo educativo de la PUCP); (ii) nivel mesocurricular (la ruta formativa que se va a seguir para la consecución de las metas formativas; se manifiesta en el plan de estudios, con sus diversas áreas, cursos obligatorios, electivos, integradores, seminarios); y (iii) nivel microcurricular (referido al diseño de cada uno de los cursos que se imparten, por lo que se manifiesta principalmente en los sílabos y cobra vida en el aula) (Carrillo y Gálvez, 2017, p. 19). 
complejo del entramado de la relación educativa que se genera entre el profesor o profesora y los y las estudiantes en el aula de la Facultad de Derecho de la PUCP; así como las labores de coordinación que entre docentes del mismo curso y los docentes de la misma área deben efectuarse.

\subsection{La posible superposición de contenidos conceptuales con otros cursos}

Se ha identificado hasta dos unidades temáticas de IDP que, potencialmente, se superponen con otros cursos dentro del Nuevo Plan. En primer lugar, se encuentra la unidad temática denominada Introducción a las Instituciones del Derecho Privado. Dicha unidad temática no es aludida de forma directa en la sumilla del Curso, pero se puede establecer una vinculación con el extremo que se refiere a los principios y fuentes del derecho civil. Lamentablemente, el sílabo del curso no permite establecer con precisión el alcance de esta unidad temática. En efecto, si se revisan los resultados de aprendizaje del curso que podrían ser de aplicación, se alude, indistintamente, a los siguientes: (i) conoce las fuentes del derecho civil; y (ii) conoce las características, naturaleza, funcionamiento y límites de las instituciones que regulan las relaciones entre privados, especialmente lo concerniente a las materias abordadas en el curso.

Esta indeterminación inicial produce una primera posible superposición con el curso de Introducción a las Ciencias Jurídicas ${ }^{14}$ (en adelante, ICJ). Si se revisa la sumilla ${ }^{15}$ y el sílabo de este curso, las Unidades Temáticas son las siguientes: (i) aproximación al concepto de Derecho (el concepto de Derecho), (ii) la norma y el sistema jurídico (concepto de norma jurídica, distinción entre reglas y principios, concepto de sistema jurídico, concepto de validez normativa, concepto de fuente de Derecho, concepto de antinomia normativa, concepto

14 Puede accederse al sílabo en el siguiente enlace: https://internacionalizacion.pucp.edu.pe/ wp-content/uploads/2016/10/6-DER243-Introducci\%c3\%b3n-a-las-Ciencias-Jur\%c3\%addicas. pdf

15 Textualmente, la sumilla indica lo siguiente: "Introducción al Derecho es un curso de formación integral y se imparte en el primer ciclo de la carrera. Busca que el estudiante aplique la metodología de resolución de casos al enfrentarse a un problema jurídico, utilizando los diversos métodos de interpretación jurídica, con lo cual contribuye al logro de las competencias excelencia académica y lógica jurídica. Es un curso que combina sesiones teóricas con prácticas donde se trabajan casos concretos. Estas se distribuyen semanalmente en cuatro horas, dos a cargo del profesor y dos asignadas a un jefe de práctica. Los temas que comprende son: el Derecho y su relación con la justicia, origen y rol en la sociedad, fuentes, principios generales y las características de la pluralidad jurídica. Asimismo, desarrolla el método de resolución de casos, interpretación e integración jurídica, considerando el análisis interdisciplinario del problema jurídico". 
de laguna normativa), y (iii) la aplicación del Derecho (ámbito temporal de la aplicación de las normas jurídicas, concepto y métodos de la interpretación jurídica, distinción entre subsunción de reglas y ponderación de principios e integración jurídica).

Tal como se puede apreciar, la superposición se produce con relación a los extremos de las Unidades Temáticas vinculadas a los principios y las fuentes del Derecho ${ }^{16}$. Frente a ello, se podría considerar que la superposición es más aparente que real, ya que mientras en ICJ se trata de los principios y las fuentes del Derecho en general; en cambio, en IDP se abordaría los "principios del Derecho privado" y las "fuentes del Derecho privado". Como soporte de esta afirmación se podría recurrir a los resultados de aprendizaje de ICJ, entre los cuales se advierten los siguientes: (i) define fuente de derecho; (ii) clasifica las fuentes del derecho; $y$, (iii) describe la jerarquía y competencia de las fuentes del derecho.

Pues bien, en opinión del autor, el intento de explicación apenas esbozado no es auspiciable, ya que dicha justificación estaría desconociendo de plano el factor histórico que alimenta los contenidos conceptuales en la enseñanza del Derecho privado. No se debe perder de vista que el tratamiento de las fuentes del Derecho en general se encuentra originariamente vinculado al estudio del Derecho privado. En esa línea se ha precisado lo siguiente:

Hay que advertir, sobre todo, que el ordenamiento didáctico de nuestras Facultades de Derecho se remonta al siglo XIX, es decir, a una época en que el derecho privado expresaba los más diversos valores políticos. El derecho privado es el derecho por excelencia; fija los principios de la propiedad privada y de la libre circulación de los bienes, de la sucesión por causa de muerte y de la obligatoriedad del contrato: tiene, en resumen, un contenido constitucional, que es presupuesto de todas las demás disciplinas jurídicas. No resulta extraño, por ello, que el derecho privado tenga, justamente, la tarea de elaborar nociones generales, que luego serán utilizadas en las materias específicas (de aquí la fusión que tuvo lugar en un período de nuestra historia universitaria, del curso de "enciclopedia jurídica", o de "introducción a las ciencias jurídicas", con el curso de "instituciones de derecho civil") (Irti, 2003, pp. 25-26).

16 Una muestra se aprecia en un autor que ha abordado, en diferentes momentos, temáticas vinculadas a la Introducción a las Ciencias Jurídicas y al Derecho Privado. Así, por ejemplo, no pasará desapercibido para ningún lector las comunes referencias, en lo que a parte del contenido se refiere, en los siguientes trabajos del profesor Marcial Rubio. Por un lado, tenemos a "El Sistema Jurídico. Introducción al Derecho" (2018) y “El Título Preliminar del Código Civil” (2018). 
Bajo esa perspectiva, entonces, se comprende que para un curso de IDP se pretenda abordar, y efectivamente se aborde, instituciones tales como la "derogación de la ley", "aplicación de las normas en el tiempo", "integración jurídica por analogía", "integración jurídica por aplicación de principios generales del Derecho" o "lagunas jurídicas". Todos estos temas son tratados por nuestra normativa civil, en concreto por el Título Preliminar del $\mathrm{CC}^{17}$. Adicionalmente, confirma lo previamente indicado, la revisión de manuales foráneos (Masi, 2004, pp. 1505-1513) y (los pocos) nacionales de Derecho privado (Espinoza, 2015; León, 2019), para advertir que dichos temas forman parte esencial del diseño de un curso institucional de introducción al Derecho privado.

En segundo lugar, se encuentra la Unidad Temática denominada por el sílabo de IDP como "El concebido y la persona natural". En específico resultará problemático el tema "Derechos de la persona". Nuevamente, estamos ante una indeterminación de los subtemas específicos a desarrollar en relación a este tópico; sin embargo, a partir del contenido del CC y de los apuntes revisados, con mayor o menor detalle, se aborda el alcance doctrinario, legislativo y jurisprudencial de los siguientes derechos de la personalidad: (i) derecho a la igualdad, (ii) el derecho a la vida, (iii) derecho a la integridad, (iv) derecho a la intimidad, (v) el derecho al honor y a la buena reputación, (vi) derecho a la imagen, (vii) los derechos de autor, entre otros.

Esta situación genera una nueva posible superposición con los contenidos de otro curso: "Derechos Fundamentales e Interpretación constitucional"18 (en adelante, DFIC), ya que según su sumilla" ${ }^{19}$ se abordan las "instituciones

17 La superposición se confirma si se presta atención a los resultados de aprendizajes de ICJ, entre los cuales se señalan los siguientes: (i) distingue, en su interrelación jerárquica, las distintas fuentes - normativas y no normativas - del sistema jurídico peruano; (ii) caracteriza los principios generales del Derecho; (iii) caracteriza, con mucha profundidad, los distintos métodos de interpretación jurídica y de integración jurídica (presupuestos, límites, ámbito de aplicación); y, (iv) emplea los métodos de interpretación e integración jurídica, relacionándolos entre sí.

18 Puede accederse al sílabo en el siguiente enlace: https://internacionalizacion.pucp. edu.pe/wp-content/uploads/2016/10/12-DEE-218-Derechos-Fundamentales-e-Interpretaci\%C3\%B3n-Constitucional.pdf

19 La sumilla indica lo siguiente: "El curso Derechos fundamentales e interpretación constitucional es un curso especializado de derecho constitucional y se imparte en el segundo ciclo de la carrera. Combina sesiones teóricas y prácticas. Busca que el alumno conozca el contenido y rol de los derechos fundamentales y domine los métodos de interpretación constitucional. De este modo, contribuye a las competencias marco ético de la profesión, excelencia académica, relación con el entorno profesional y paradigma del estado constitucional de derecho. El curso estudia las instituciones jurídicas, teorías, jurisprudencia y doctrina nacional e internacional de los derechos fundamentales; su rol en el ordenamiento jurídico y la economía social de mercado; 
jurídicas, teorías, jurisprudencia y doctrina nacional e internacional de los derechos fundamentales; su rol en el ordenamiento jurídico y la economía social de mercado; el contenido de derechos fundamentales esenciales, como el derecho a la vida, libertad, honor y libertad de expresión"20.

En este caso, es la comparación jurídica la que puede brindar una explicación de la posible superposición. Y es que gracias a aquella se conoce que la regulación actual de nuestro $\mathrm{CC}$, en materia de derecho de las personas, tuvo una influencia directa del Código Civil italiano (en adelante, Codice), el cual responde a una tradición cultural (la europea) en la que el reconocimiento de los derechos de la personalidad, de forma originaria, se dio a través de los códigos civiles, siendo recién posteriormente reconocidos por textos constitucionales (León, 2004, p. 279). En esa línea se ha precisado que:

En el modelo de constitucionalismo europeo-continental, de matriz francesa, la positivización de los derechos fundamentales no produjo una codificación del tipo de la Constitución americana- la primer gran codificación moderna- por el contrario el código civil, es decir la codificación de las libertades burguesas y de las relaciones de libre mercado en la forma de derecho civiles privados (Mengoni, 2011b, p. 319).

Sin embargo, la tradición cultural europea, que en la actualidad permite a su doctrina reflexionar respecto del valor constitucional de la codificación (CabriIlac, 2003), en este extremo no es similar a la peruana, ni a la Latinoamericana en general, en donde se tiene como idea asentada el valor vinculante y superior de los textos constitucionales respecto de los demás productos normativos.

Entonces, surge la duda legítima si este último hecho es un justificativo determinante para inclinar la balanza, ahora desde una perspectiva curricular, a favor de que el tratamiento de los derechos de la personalidad se reserve

el contenido de derechos fundamentales esenciales, como el derecho a la vida, libertad, honor y libertad de expresión; y los principios de interpretación constitucional, profundizando en el test de proporcionalidad". Esta sumilla se encuentra disponible en: http://facultad.pucp.edu.pe/ derecho/wp-content/uploads/2019/03/Sumillas-de-los-cursos-obligatorios.WEB_.pdf.

20 Nuevamente, esto se confirma si se revisa los resultados de aprendizaje de ICJ entre los que menciona los siguientes: (i) identifica las situaciones en las que pudiera existir algún conflicto o vulneración de derechos fundamentales, (ii) explica algunos rasgos de la evolución histórica de las instituciones jurídicas nacionales de los derechos fundamentales, (iii) caracteriza la doctrina y jurisprudencia nacional e internacional más importante sobre los derechos fundamentales, (iv) explica los derechos fundamentales en el marco del modelo de economía social de mercado, (v) identifica teorías de los derechos fundamentales; y, (vi) define y distingue la naturaleza, titularidad, contenido y límites de los derechos fundamentales. 
exclusivamente al ámbito de los cursos de Derecho constitucional. En opinión del autor, ese no es el camino a seguir.

\subsection{El posible no tratamiento de contenidos conceptuales propios de IDP}

De forma conjunta con la situación anterior, en la que parte de los contenidos conceptuales del curso de IDP se podían superponer a los contenidos de otros cursos, es posible identificar escenarios específicos en los que los contenidos conceptuales de IDP no son desarrollados de forma uniforme por los profesores y profesoras a cargo de dicho curso.

En primer lugar, tal como se aprecia en la sumilla del curso de IDP, una de las Unidades Temáticas es "Situaciones Jurídicas", la cual sí tiene un detalle de los temas que comprende. En específico se señalan los siguientes: (i) concepto y clasificación de las situaciones y relaciones jurídicas, (ii) principales instituciones de derecho privado que generan situaciones y relaciones jurídicas (actos jurídicos, contratos, actos ilícitos, relaciones familiares, derechos reales y herencia), (iii) importancia de los Registros Públicos en la protección de los derechos derivados de situaciones y relaciones jurídicas.

La inclusión expresa del tema Situaciones Jurídicas en IDP responde a una necesidad específica generada por el antiguo Plan. Bajo la malla curricular anterior podía configurarse dos situaciones: (i) nunca haber abordado la problemática de las Situaciones Jurídicas o (ii) repetir el mismo contenido en más de un curso, siempre de Derecho Patrimonial, a lo largo de la carrera universitaria ${ }^{21}$.

Sin perjuicio de lo anterior, la importancia del desarrollo del tema de Situaciones Jurídicas es innegable. El manejo de nociones como derecho subjetivo, derecho potestativo, facultad, poder, expectativa, deber, estado de sujeción, carga, entre otras resulta fundamental para el Derecho privado en particular y para el Derecho en general. Permite establecer una suerte de lenguaje jurídico (Zatti, 2005, p. 357; Fernández, 1994, pp. 41-42) de suma utilidad, siempre que no se olvide su valor convencional (Roppo, 2013, pp. 58-59); y, sobre todo, establece las bases para un adecuado diseño de un sistema de mecanismos de protección jurídica o de tutela jurídica sustancial.

Sin embargo, de la constatación de los apuntes de los alumnos y alumnas que han llevado IDP se pueden apreciar dos tendencias muy marcadas: (i) no

21 A modo de ejemplo, y como testimonio personal del autor, en cuatro de los cursos de pregrado de Facultad (Acto Jurídico con Rómulo Morales, Contratos: Parte General con Hugo Forno, Contratos Típicos con Jorge Beltrán y Responsabilidad Civil con Gastón Fernández) se desarrolló el tema de Situaciones Jurídicas, aunque siempre bajo diversas perspectivas. 
se desarrolla el concepto y clasificación de las situaciones jurídicas subjetivas, limitándose a la descripción general de los hechos generadores de situaciones y relaciones jurídicas y (ii) se aborda el concepto y clasificación de las situaciones jurídicas subjetivas de forma institucional, así como una presentación de los hechos jurídicos que las originan.

A modo de hipótesis, se puede sugerir que la primera tendencia se explica por el hecho de que no se ha interiorizado la modificación de los contenidos conceptuales de IDP respecto de su equivalente en el antiguo Plan (es decir, Derecho Civil 1: PP.GG. y PP.NN.), el cual no contemplaba el tema de Situaciones Jurídicas.

En segundo lugar, otra de las Unidades Temáticas de IDP es "La persona jurídica como sujeto de derecho". El detalle específico de esta unidad comprende los siguientes temas: (i) concepto, reconocimiento, clases, finalidad, derechos, autonomía patrimonial y capacidad, (ii) responsabilidad civil de la persona jurídica, (iii) cambios en la persona jurídica, (iv) asociación, fundación y comité, y (v) las organizaciones no inscritas.

Que se aborde este tema en IDP es una necesidad, ya que el Nuevo Plan, adecuadamente, ya no considera el curso "Personas Jurídicas" como uno de carácter obligatorio. En ese sentido, el no abordar este tema no solo afecta a los cursos que pertenecen a la rama de Derecho Civil, sino a todos los cursos en general, ya que, desde Derecho Constitucional hasta Derecho Contable, pasando por Derecho Mercantil, que son cursos que prosiguen dentro de la malla curricular, requieren de los fundamentos del régimen jurídico general de las Personas Jurídicas.

No obstante la importancia de lo apenas indicado, el tratamiento que se le da a la Unidad Temática "Personas Jurídicas" es del todo irregular presentándose hasta tres tendencias: (i) no se desarrolla la teoría general de las personas jurídicas y solo se recurren a ejemplos puntuales, (ii) se desarrolla la teoría general de las personas jurídicas de forma aproximativa, sin entrar a la problemática de las personas jurídicas sin fines de lucro, y (iii) se desarrolla de forma institucional la problemática de las personas jurídicas haciendo referencia, inclusive, al V Pleno Casatorio Civil (que se basó en la nulidad de los negocios jurídicos colegiales de las personas jurídicas sin fines de lucro) y al tratamiento registral de las personas jurídicas no lucrativas.

En este caso, al igual que en el supuesto anterior, una posible explicación del tratamiento tan dispar de la teoría general de las personas jurídicas se debe a considerar que dicho tema es materia de otro curso, lo cual ya no es así en el Nuevo Plan. Si bien existe un curso optativo, denominado, Personas Jurídicas; 
su alcance es diferente, ya que no supone la presentación de la institución, sino la aplicación integradora de saberes previos.

A continuación, de forma meramente referencial, se esbozan algunas sugerencias para poder ayudar a rediseñar los contenidos conceptuales de IDP.

\section{SUGERENCIAS PARA UN REDISEÑO DE LOS CONTENIDOS CONCEP- TUALES DEL CURSO IDP}

Dado que la superposición y el no desarrollo de temas han sido las dos principales observaciones que se han esbozado, corresponde ahora formular algunas sugerencias para enfrentarlas. Estas no tienen, y no podrían tener, una vocación de imperatividad, sino tan solo pretenden ser propuestas de alternativas que provoquen en los y las docentes a cargo de IDP una labor de introspección que lleve a la evaluación del diseño (y ejecución) de los contenidos conceptuales del curso IDP.

\subsection{Alternativas para eliminar la superposición de contenidos concep- tuales del IDP con otros cursos del Nuevo Plan}

En relación con el primer punto (superposición entre IDP e ICJ), hay que precisar que, si se admite que la temática vinculada a la teoría de la norma jurídica es propia del Derecho Privado, entonces la natural conclusión a la que debe arribar es la eliminación de dichos tópicos del curso de ICJ. Con ello se reservaría para este curso (quizá) temas vinculados a la Filosofía del Derecho, tales como el concepto de Derecho o la noción de justicia. Lo cual, no obstante, no está libre de problemas, ya que deberá realizarse una fina adecuación entre estos cursos y el de Argumentación Jurídica, por ejemplo, todo con el objetivo de que el estudiante no repita los mismos temas, más allá de lo mínimo necesario, a lo largo de su formación universitaria.

Sin embargo, la posición de la Facultad de Derecho de la PUCP parece haber tomado partido por mantener la vigencia del curso de ICJ con tanto énfasis que es el único curso que cuenta con un horario específicamente previsto para el desarrollo de Jefaturas de Prácticas. Dicha toma de partido, entonces, debe ser coherente a lo largo del diseño de los cursos de la nueva malla curricular.

Y es que apostar por un curso general de ICJ, antes que vaciar de contenido al curso de IDP, conlleva un reconocimiento de la utilidad de perspectivas que permitan la generalización del conocimiento. Generalización que es perfectamente aplicable, y por ende defendible, en otros cursos del área del Derecho Privado. Entonces, implicaría una contradicción manifiesta, por ejemplo, re- 
forzar el curso de ICJ, pero no prestar la misma atención a IDP o, para ser más enfático, reducir la importancia de un curso como IDP2.

En este contexto, la sugerencia que se formula no es prescindir del desarrollo del contenido conceptual acerca de la "teoría general de la norma jurídica", sino que se aborde desde una perspectiva histórica (Larenz, 2001, pp. 177-328), comparada (Vogenauer, 2019, pp. 877-901; Sacco y Gambaro, 2018, pp. 31-45 y 169-196) y, claro está, aplicada, que permita efectuar, previo reconocimiento de una metodología del Derecho Privado (Canaris, 1995, pp. 25-70; Zimmermann, 2019, pp. 241-287), una labor crítica respecto de postulados que, tradicionalmente, se afirman sin mayor reflexión, tales como alguno de los siguientes: la jurisprudencia es fuente de derecho, la doctrina es fuente de derecho, las normas solo pueden ser reglas o principios, el conflicto entre principios se resuelve únicamente a través de la ponderación. Esta aproximación permitiría desarrollar las Competencias Específicas de Derecho vinculadas a la Excelencia Académica.

Con relación al segundo punto (superposición entre IDP y DFIC), la problemática se puede presentar de forma manifiesta con relación a los contenidos conceptuales de ambos cursos. Claro está, ello dependerá de la forma cómo se aborden dichos contenidos conceptuales en el curso de IDP. Si él o la docente brinda una importancia central a los derechos de la personalidad, entonces aumenta la probabilidad que el alumno o alumna vea duplicada una parte sustancial de dicho contenido en el curso de DFIC. Por el contrario, si la aproximación a los derechos de la personalidad es delimitada, entonces antes que una superposición se podrá establecer un complemento entre ambos cursos.

Nuevamente, aquí se debe conciliar esta perspectiva con la visión global que la Facultad de Derecho ha asumido con relación al paradigma del Estado Constitucional de Derecho, a tal nivel que incluso aparece expresamente indicado como una de las Competencias Específicas de Derecho. Así las cosas, todo indicaría que los cursos de Derecho Constitucional tienen un fuero de atracción respecto de los temas vinculados a los derechos de la personalidad y su protección, siempre en el fuero constitucional.

Desde el punto de vista del autor, la lectura previamente expuesta, puede terminar siendo reduccionista, porque no toma en consideración que los mecanismos de protección jurídicos de los derechos de la personalidad trascienden del ámbito constitucional, encontrando en el Derecho Privado un precioso espacio para su desarrollo (León, 2006, pp. 11-19; Fernández, 2017, pp. 21-44) 
que debe ser puesto a consideración de los alumnos y alumnas de la Facultad de Derecho de la PUCP.

En este contexto, los derechos de la personalidad claro que deben ser materia de estudio en IDP. Solo que su concreto alcance debe estar adecuadamente delimitado. Bajo esta premisa, se sugiere que en IDP se aborde el complicado problema de la relación entre la autonomía de los particulares y los derechos de la personalidad (Mak, 2008; Nicolussi, 2016, pp. 119-143). Ello supone una presentación inicial de lo que es un derecho de la personalidad, para inmediatamente abordar su relación con la autonomía de los particulares vinculada al poder de disposición de este tipo de situaciones jurídicas subjetivas. En este marco de referencia, resultan revisitados temas como negocios jurídicos respecto del nombre, actos de disposición del propio cuerpo, consentimiento informado para tratamientos médicos, negocios de explotación respecto de derechos de autor, entre otros.

\subsection{Alternativas para el desarrollo de los contenidos conceptuales pre- vistos en IDP}

Veamos ahora los alcances dentro de los que pueden ser abordadas las Situaciones Jurídicas y las Personas Jurídicas para que su desarrollo pueda tener un mínimo grado de homogeneidad en IDP.

En lo que concierne a las Situaciones Jurídicas el contenido mínimo (Breccia et al., 1992, pp. 365-532; y desde una perspectiva constitucional, Perlingieri, 2015, pp. 749-1175) a ser desarrollado puede ser el siguiente: (i) concepto de situación jurídica subjetiva, (ii) clasificación de situación jurídica subjetiva (situaciones de ventaja, de desventaja, activas y pasivas), (iii) contenido de las principales situaciones jurídicas subjetivas (poder, facultad, derecho subjetivo, derecho potestativo, expectativa, interés legítimo, deber, carga), (iv) ejercicio de las situaciones jurídicas subjetivas (límites al ejercicio de derecho, conflicto de derechos, abuso de derecho), (v) vicisitudes de las situaciones jurídicas subjetivas (creación, modificación, regulación y extinción), y (vi) introducción a los mecanismos de tutela jurídica sustancial (inhibitoria, remoción, resarcimiento).

Abordar los temas apenas mencionados es de vital importancia. Por ejemplo, con miras a IDP2, la estructura propuesta de IDP permite realizar una adecuada conexión con el estudio de la "Prescripción y caducidad" que son propios de la Parte General del Derecho privado. Ni qué decir con relación a los cursos de Contratos, Obligaciones y Responsabilidad civil. Con atención a los cursos del área de Derecho Procesal, su utilidad es tremenda, ya que ello 
permitirá una mejor aproximación a la noción de pretensión, tutela jurisdiccional, carga de la prueba, poderes, entre otros. Los cursos propios del área del Derecho Administrativo contarían con mayores elementos de juicio para distinguir categorías propias del Derecho Público en comparación con las de Derecho Privado y develar lo inexacto de aproximaciones tradicionales como la afirmación que el Estado cuenta con poderes exorbitantes cuando contrata, en la medida que ello también es propio de las relaciones de naturaleza privada.

Finalmente, en lo que respecta al desarrollo de las Personas Jurídicas, el contenido mínimo (Ferrara, 2006; Falzea y Basile, 1983) que se sugiere que sea abordado es el siguiente: (i) noción de persona jurídica (separación afirmativa y negativa de activos, constitución, levantamiento del velo), (ii) capacidad de las personas jurídicas (toma de decisiones, consecuencias en la responsabilidad de las personas jurídicas), (iii) titularidad de situaciones jurídicas por parte de las personas jurídicas (problemática respecto de la titularidad de derechos fundamentales), (iv) objeto de la persona jurídica (problemática referida a los actos ultra vires), (v) clasificación de personas jurídicas (lucrativas y no lucrativas), (vi) tipología de personas jurídicas no lucrativas (asociación, fundación y comité), (vii) extinción de las personas jurídicas no lucrativas, y (viii) transformación de las personas jurídicas no lucrativas.

El desarrollo de esta temática es determinante ya que es el único curso en donde se podrán desarrollar los temas apenas mencionados. Adicionalmente, representa un insumo directo para cursos posteriores tales como IDP2, Derechos Reales, Derecho Contable, Instituciones del Derecho Mercantil, Derechos Fundamentales e interpretación constitucional, entre otros.

\section{REFLEXIÓN FINAL: EL CARÁCTER PROPEDÉUTICO DE LAS INSTITU- CIONES DEL DERECHO PRIVADO}

En el Nuevo Plan, la importancia de IDP es fundamental. El curso de IDP, junto con IDP2, está diseñado para construir la Parte General del Derecho Privado. Una temática que tiene un carácter propedéutico dentro de la formación de todo alumno o alumna de la Facultad de Derecho de la PUCP.

En ese sentido, los contenidos conceptuales de IDP se han reconfigurado en comparación con el curso de Derecho Civil 1. Ello determina que las y los docentes a cargo de este curso se encuentren ante el desafío de adecuarse a la apuesta formativa que la Facultad de Derecho ha realizado al desarrollar cursos con ámbitos de saberes generalizantes. 
Parte del desafío antes indicado conlleva la evaluación transversal de los contenidos conceptuales de IDP con los otros cursos que conforman la malla curricular, ello con el ánimo de buscar y consolidar una coherencia interna dentro del Nuevo Plan y, a nivel macro, con el Modelo Educativo de la PUCP.

\section{RECOMENDACIONES: PROPUESTA DE CONTENIDOS CONCEPTUALES PARA EL CURSO IDP}

Para concluir esta breve reflexión, siempre a nivel de sugerencia y con el solo ánimo de contribuir a la mejora de los contenidos conceptuales de IDP para un debate posterior, se presenta, a continuación, una propuesta de sistematización de los mismos, en atención a la problemática abordada a lo largo del texto:

\begin{tabular}{|l|l|}
\hline \multicolumn{1}{|c|}{ UNIDAD TEMÁTICA } & \multicolumn{1}{|c|}{ CONTENIDOS CONCEPTUALES } \\
\hline Principios y fuentes del Derecho civil & $\begin{array}{l}\text { 1. } \begin{array}{l}\text { Derecho privado (concepto y relación } \\
\text { con el Derecho Público). }\end{array} \\
\text { 2. Ámbito de actuación del Derecho pri- } \\
\text { vado. }\end{array}$ \\
\begin{tabular}{|l|l} 
3. $\begin{array}{l}\text { Metodología del Derecho privado des- } \\
\text { de una perspectiva histórica. }\end{array}$ \\
4. Fuentes de Derecho (aproximación \\
histórica).
\end{tabular} \\
\begin{tabular}{|l} 
5. $\begin{array}{l}\text { Codificación (aproximación de derecho } \\
\text { comparado). }\end{array}$ \\
6. Constitucionalización del Derecho pri- \\
vado. \\
7. $\begin{array}{l}\text { Norma jurídica privatista (lenguaje y } \\
\text { clasificación). }\end{array}$ \\
8. Introducción a los hechos jurídicos \\
(hechos jurídicos en sentido estricto, \\
actos jurídicos en sentido estricto y \\
negocio jurídico).
\end{tabular} \\
\hline
\end{tabular}




\begin{tabular}{|c|c|}
\hline Situaciones jurídicas & $\begin{array}{l}\text { 1. Concepto de situación jurídica subjetiva } \\
\text { 2. Clasificación de situación jurídica } \\
\text { subjetiva (situaciones de ventaja, de } \\
\text { desventaja, activas y pasivas). } \\
\text { 3. Contenido de las principales situa- } \\
\text { ciones jurídicas subjetivas (poder, } \\
\text { facultad, derecho subjetivo, derecho } \\
\text { potestativo, expectativa, interés legíti- } \\
\text { mo, deber, carga). } \\
\text { 4. Ejercicio de las situaciones jurídicas } \\
\text { subjetivas (límites al ejercicio de dere- } \\
\text { cho, conflicto de derechos, abuso de } \\
\text { derecho). } \\
\text { 5. Vicisitudes de las situaciones jurídicas } \\
\text { subjetivas (creación, modificación, } \\
\text { regulación y extinción). } \\
\text { 6. Introducción a los mecanismos de } \\
\text { tutela jurídica sustancial (inhibitoria, } \\
\text { remoción, resarcimiento). }\end{array}$ \\
\hline Personas naturales & $\begin{array}{l}\text { 1. Existencia de la persona natural } \\
\text { (nacimiento, muerte, desaparición, } \\
\text { ausencia, muerte presunta). } \\
\text { 2. La tutela jurídica del concebido (pro- } \\
\text { tección jurídica del embrión, técnicas } \\
\text { de reproducción humana asistida). } \\
\text { 3. Identificación de la persona natural } \\
\text { (nombre, localización y publicidad } \\
\text { registral). } \\
\text { 4. Capacidad de la persona natural (visión } \\
\text { tradicional de la capacidad jurídica, im- } \\
\text { pacto de la Convención de los Derechos } \\
\text { de las Personas con Discapacidad) } \\
\text { 5. Derechos de la personalidad (contenido } \\
\text { esencial de los derechos fundamenta- } \\
\text { les, limitación por la autonomía de os } \\
\text { particulares) }\end{array}$ \\
\hline
\end{tabular}


Personas jurídicas

Tabla 2. Fuente: Elaboración propia.
1. Noción de persona jurídica (separación afirmativa y negativa de activos, constitución, levantamiento del velo).

2. Capacidad de las personas jurídicas (toma de decisiones, consecuencias en la responsabilidad de las personas jurídicas).

3. Titularidad de situaciones jurídicas por parte de las personas jurídicas (problemática respecto de la titularidad de derechos fundamentales).

4. Objeto de la persona jurídica (problemática referida a los actos ultra vires).

5. Clasificación de personas jurídicas (lucrativas y no lucrativas).

6. Tipología de personas jurídicas no lucrativas (asociación, fundación y comité).

7. Extinción de las personas jurídicas no lucrativas.

8. Transformación de las personas jurídicas no lucrativas.

\section{LISTAS DE REFERENCIA}

Adam, S. (2006). An introduction to learning outcomes. A consideration of the nature, function and position of learning outcomes in the creation of the European Higher Education Area. https://is.muni.cz/do/1499/metodika/ rozvoj/kvalita/Adam_IH_LP.pdf

Breccia, U, Bigliazzi, L., Natoli, U. y Busnelli, F. (1992). Derecho civil, t. I, vol. 1. Universidad Externado de Colombia.

Cabrillac, R. (2003). ¿Es el Code civil la verdadera Constitución de Francia? (José Gabriel, trad.). Themis, 63.

Canaris, C. (1995). Función, estructura y falsación de las teorías jurídicas (Daniela Brückner y José Luis de Castro, trads.). Civitas.

Carrillo, G. y Gálvez, A. (2017). Formación por competencias. En Colección Métodos de Formación Jurídica, 1. Facultad de Derecho de la Pontificia Universidad Católica del Perú. 
Espinoza, J. (2015). Introducción al Derecho Privado. Los principios contenidos en el Título Preliminar del Código Civil. Análisis doctrinario, legislativo y jurisprudencial, 4 ed. Actualidad Jurídica, 1, 73-80. Instituto Pacífico.

Falzea, A. y Basile, M. (1983). Persona giuridica (diritto privato). Giuffrè.

Fernández Cruz, G. (1994). La obligación: apuntes para una dogmática jurídica del concepto. Themis, 27-28, 41-56.

Fernández Cruz, G. (2017). La cláusula penal. Tutela contra el incumplimiento vs. tutela resarcitoria. Ara.

Ferrara, F. (2006). Teoría de las personas jurídicas. Comares.

Genty, P. (2018). Celebrating the difference. A U.S. educator's perspective on legal education under the civil and common law. En R. Grimes (ed.), Re-thinking legal education under the civil and common law, A road map for constructive change. https://doi.org/10.4324/9781315212074

Irti, N. (2003). Introducción al estudio del Derecho privado, (Rómulo Morales y Leysser León, trads. y notas). Grijley.

Kennedy, D., Hyland, Á. y Norma, R. (2007). Writing and using learning outcomes: a practical guide. http://www.procesbolonski.uw.edu.pl/dane/learning-outcomes.pdf

Larenz, K. (2001). Metodología de la ciencia del Derecho. Ariel.

León, L. (2004). El sentido de la codificación civil. Estudios sobre la circulación de los modelos jurídicos y su influencia en el Código Civil peruano. Palestra.

León, L. (2006). Derecho a la intimidad y responsabilidad civil: el refuerzo de los derechos fundamentales a través de los remedios civilísticos. En Cuaderno de Trabajo, 2. Departamento Académico de Derecho.

León, L. (2012). Retos de la enseñanza del Derecho Civil, Jurídica, 8, 437.

León, L. (2019). Derecho privado. Parte general. Negocios, actos y hechos jurídicos. En Colección lo esencial del Derecho. Fondo editorial de la Pontificia Universidad Católica del Perú.

Lipari, N. (2004). Sull'insegnamento del Diritto civile. En V. Scalisi (ed.), Scienza e insegnamento del diritto civile in Italia. Convegno di studio in onore del prof. Angelo Falzea (Messina, 4-7 giugno 2002). Giuffrè.

Mak, C. (2008). Fundamental rights in European Contract Law. A comparison of the impact of fundamental rights on contractual relationships in Germany, the Netherlands, Italy and England. https://doi.org/10.1017/9781780684963

Masi, A. (2004). L'ampiezza dei manuali istituzionali di diritto civile e di diritto privato. En V. Scalisi (ed), Scienza e insegnamento del diritto civile in Italia. 
Convegno di studio in onore del prof. Angelo Falzea (Messina, 4-7 giugno 2002). Giuffrè.

Mendoza, G. (2018). El génesis de la Parte General del Bürgerliches Gesetzbuch. Themis, 73. https://doi.org/10.18800/themis.201801.008.

Mengoni, L. (2011a). L'insegnamento del Diritto nell'Università Cattolica. En C. Castronovo, A. Albanese y A. Nicolussi (eds.), Scritti I: Metodo e teoria giuridica. Giuffrè.

Mengoni, L. (2011b). L'Europa del codici o un codice per l'Europa? En C. Castronovo, A. Albanese y A. Nicolussi (eds.), Scritti I: Metodo e teoria giuridica. Giuffrè.

Merryman, J. (1975). Legal education in civil law and common law universities: a comparison of objectives and methods. En: L'educazione giuridica, vol. I. Università degli Studi di Perugia, Consiglio Nazionale delle Ricerche, Libreria Universitaria.

Nicolussi, A. (2016). Autonomia contrattuale e diritti della persona. En Giurisprudenza per principi e autonomia privata. Giappichelli.

Morales, R. (2010). Educación jurídica y Derecho Civil. Una propuesta de reforma académica. Foro jurídico, 10, 318-321.

Perlingieri, P. (2015). El Derecho Civil en la legalidad constitucional, vol. II. Pontificia Universidad Javeriana.

PUCP. (2014). Guía para la elaboración del Plan de Estudios de Pregrado. Fondo editorial de la Pontificia Universidad Católica del Perú.

PUCP. (2016). Modelo Educativo PUCP. Fondo editorial de la Pontificia Universidad Católica del Perú.

Ramos, C. y Cuadros, H. (2019). Crónicas del claustro: cien años de historia de la Facultad de Derecho de la PUCP. Fondo editorial de la Pontificia Universidad Católica del Perú.

Roppo, V. (2013). Diritto privato, 3 ed. Giappichelli.

Sacco, R. y Gambaro, A. (2018). Sistemi giuridici comparati. En R. Sacco (dir.). Trattato di Diritto Comparato. Utet.

Sanromán, R. y Morales, L. (2016). La educación por competencias en el campo del derecho. Boletín Mexicano de Derecho Comparado, 19, 146). https:// doi.org/10.22201/iij.24484873e.2016.146.10510

Vogenauer, S. (2019). Sources of law and legal method in Comparative Law. En M. Reimann y R. Zimmermann (eds.), The Oxford Handbook of Comparative Law. 10.0.4.69/oxfordhb/9780198810230.001.0001 
Zatti, P. (2005). Las situaciones jurídicas, Vladimir Contreras y Gilberto Mendoza (trads.), Revista Jurídica del Perú, LV, 64.

Zimmermann, R. (2019). Juristische Methodenlehre in Deutschland. Rabels Zeitschrift für ausländisches und internationales Privatrecht, 83. https://doi. org/10.1628/rabelsz-2019-0021 